\title{
Electrically Controlled Phase Shifter using Semiconductor Laser in Optical Single Sideband System
}

\author{
F. M. Wu ${ }^{1}$, P. C. Peng ${ }^{2}$, S. K. Yeh ${ }^{1}$, C. T. Lin ${ }^{1}$, J. H. Chen ${ }^{1}$, S. Chi ${ }^{1,3}$ \\ 1. Department of Photonics and Institute of Electro-Optical Engineering, National Chiao Tung University, Hsinchu, Taiwan, R. O. C. \\ 2. Department of Electro-Optical Engineering, National Taipei University of Technology, Taipei, Taiwan, R.O.C. \\ 3. Department of Electrical Engineering, Yuan-Ze University, Chung-Li, Taiwan, R.O.C. \\ E-mail: pcpeng@ntut.edu.tw
}

\begin{abstract}
This investigation experimentally explores a microwave phase shifter using a semiconductor laser in an optical single sideband system. The operational bandwidth from 12 to $20 \mathrm{GHz}$ is achieved by adjusting the bias current.
\end{abstract}

\section{Introduction}

In recent years, microwave photonic devices have attracted much attention because of their many advantages such as immunity to electromagnetic interference, wide bandwidth, excellent isolation, small size, and low weight [1-2]. One of the key devices in microwave systems is phase shifters. Recently, photonic microwave phase shifters have been reported [2]. Moreover, tunable slow light devices and phase shifters using optical semiconductor devices also have been experimentally demonstrated [3]. However, the microwave phase shifter based on a vertical-cavity surface-emitting laser (VCSEL) in an optical single sideband system has not yet been explored.

In this paper, we experimentally explore a VCSEL for a microwave phase shifter in an optical single sideband system. Optical single sideband systems can eliminate completely the effects of fiber chromatic dispersion in radio over fiber system [4-5]. The operational frequency from 12 to $20 \mathrm{GHz}$ is achieved by adjusting the bias current of VCSEL. This investigation will be useful in microwave photonic systems.
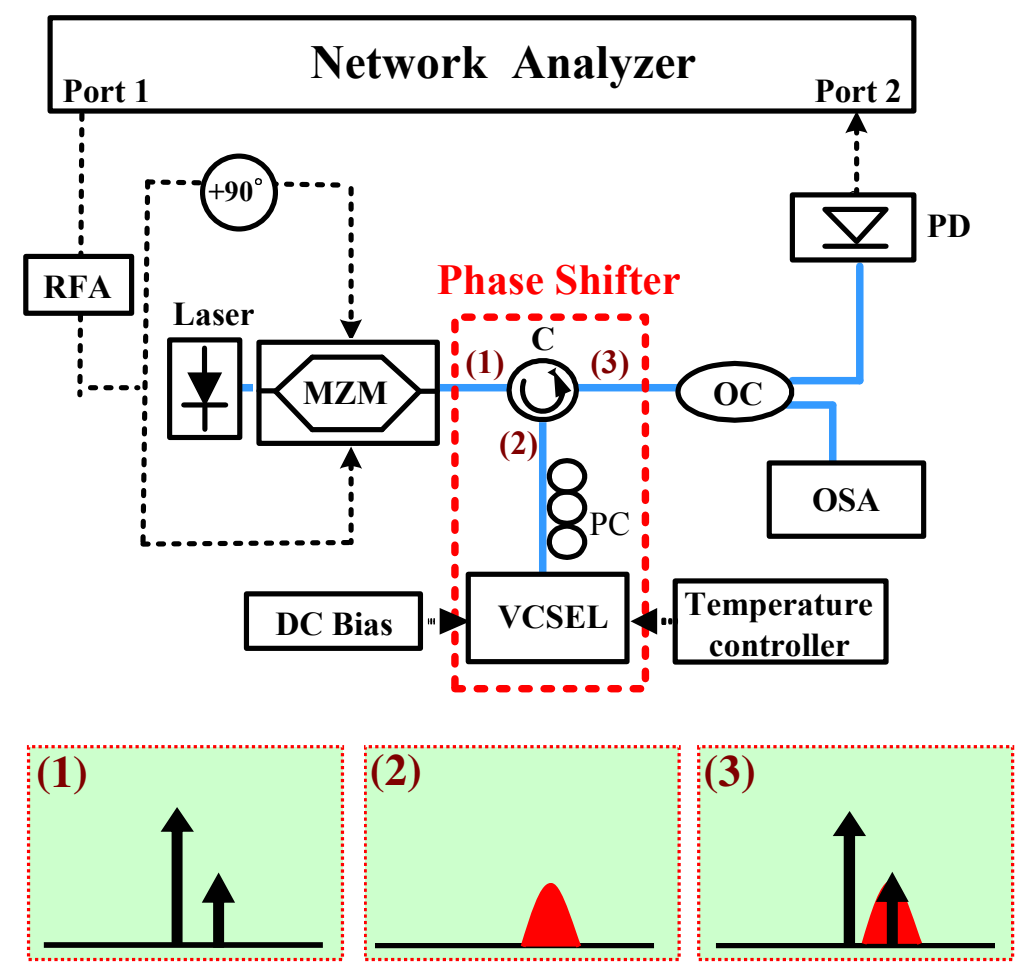

Fig. 1. Experimental setup for measuring the microwave phase shifter in the optical single sideband system. (MZM: dual-drive Mach-Zehnder Modulator, C: optical circulator, OC: optical coupler, PC: polarization controller, RFA: RF amplifier, PD: photodetector, OSA: optical spectrum analyzer) 


\section{Experiments and Results}

The experimental setup for measuring the microwave phase shifter in the optical single sideband system is schematically depicted in Fig.1. The probe signal is generated by a laser source and modulated by a dual-drive Mach-Zehnder modulator (MZM). The output spectrum of optical single sideband signal is shown in Fig. 2. The optical single sideband signal is injected into a VCSEL via an optical circulator (C). Fig. 3 shows the optical spectra of VCSEL at the various bias currents.

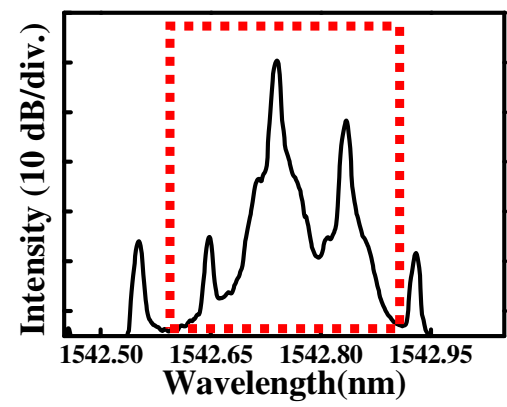

Fig. 2. Output spectrum of single sideband signal.

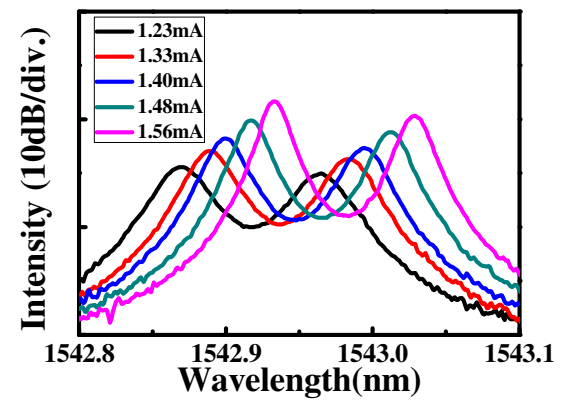

Fig. 3. Output spectra of VCSEL at the various bias currents.

The polarization controller (PC) is used to adjust the polarization of probe signal. The output light from the VCSEL is guided through the optical circulator. Than, the optical signal is split by a $1 \times 2$ optical coupler, one is sent to optical spectrum analyzer (OSA), another is sent to photodetector (PD). Finally, the amplitude and phase changes are measured by a network analyzer. In the experiment, the power of probe signal is kept constant while the bias current of VCSEL is varied. Fig. 4 shows the amplitude and phase change response of the QD VCSEL at the various bias currents. The bias currents of VCSEL in Fig. 4 are $1.23 \mathrm{~mA}, 1.33 \mathrm{~mA}, 1.40 \mathrm{~mA}, 1.48 \mathrm{~mA}$, and $1.56 \mathrm{~mA}$, respectively. When the bias currents change from $1.23 \mathrm{~mA}$ to $1.56 \mathrm{~mA}$, a continuous phase change can be achieved.

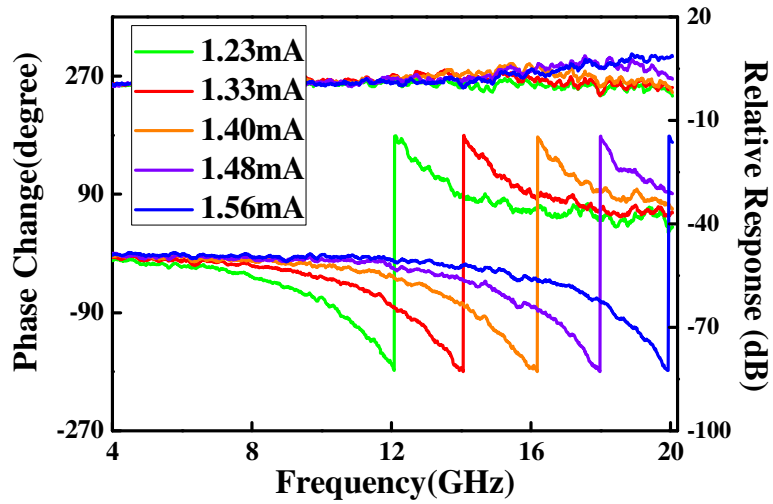

Fig. 4. Amplitude and phase change response at $1.23 \mathrm{~mA}, 1.33 \mathrm{~mA}, 1.40 \mathrm{~mA}, 1.48 \mathrm{~mA}$, and $1.56 \mathrm{~mA}$, respectively.

\section{Conclusion}

This study experimentally demonstrated the electrically controlled phase shifter based on the VCSEL in the optical single sideband system. The tunable range from 12 to $20 \mathrm{GHz}$ is achieved by adjusting the bias current from $1.23 \mathrm{~mA}$ to $1.56 \mathrm{~mA}$. The microwave phase shifter using the VCSEL has the potential to reduce the size and cost of microwave photonic systems.

\section{References}

1] J. P. Yao "Microwave photonics," Journal of Lightwave Technology, vol. 27, no. 3, pp. 314-335, 2009.

[2] E. H. W. Chan, and R. A. Minasian, "Photonic RF Phase Shifter and Tunable Photonic RF Notch Filter", Journal of Lightwave Technology, vol. 24, pp. 2676-2682,2006.

[3] C. J. Chang-Hasnain and S. L. Chuang, "Slow and Fast Light in Semiconductor Quantum-Well and Quantum-Dot Devices," Journal of Lightwave Technology, vol. 24, pp.4642-4654, 2006.

[4] G. H. Smith, D. Novak, and Z. Ahmed, "Overcoming chromatic dispersion effects in fiber-wireless systems incorporating external modulators," IEEE Trans. Microw. Theory Tech., vol. 45, no. 8, pp. 1410-1415, 1997.

[5] S. Blais and J. P. Yao, "Optical single sideband modulation using an ultranarrow dual-transmission-band fiber Bragg grating," IEEE Photonics Technology Letters, vol. 18, no. 21, pp. 2230-2232, 2006. 